\title{
The manipulation of stimulus quality and the definition of stimulus encoding operations in memory scanning experiments
}

\author{
MARY HARDZINSKI and ROBERT G. PACHELLA \\ University of Michigan, Ann Arbor, Michigan 48104
}

\begin{abstract}
Two experiments examine procedures for defining and isolating stimulus encoding processes within the standard memory scanning task. Two manipulations are used to converge on this definition: letter case (i.e., physical vs. name matching of letters) and stimulus quality. Experiment 1 produced equal scanning rates for the name match and the control conditions in which letter case was not varied. The physical match conditions produced scanning rates half as great as the control. None of these rates were greatly affected by the degradation (contrast reduction) of the probe stimulus, although the small difference in rates for the physical match condition was significant. Experiment 2 investigated two modes of stimulus degradation, contrast reduction and the addition of visual noise. All of the results of the first experiment were replicated for both modes of degradation, with the exception of the change in the scanning rates for the physical match condition. In addition, visual noise produced greater differences between positive and negative response times than did contrast reduction, which did not differ from the high contrast control condition. These results indicate that an abstract internal code is derived during the encoding of the probe stimulus from which the effects of stimulus quality have been removed. Thus, factors that interact with stimulus quality in memory scanning tasks can be assumed to have a locus within the encoding stage of processing.
\end{abstract}

Miller and Pachella (1973, 1976) and Stanovich and Pachella (1977) have demonstrated that the effect of stimulus probability on reaction time in a simple memory scanning task was greatly increased when the clarity or contrast of the probe stimulus used in the task was reduced. They interpreted this simple interaction in the context of Sternberg's (1969) method of additive factors to indicate that stimulus probability and stimulus quality had some common loci of operation within the information processing sequence. Furthermore, they concluded that one of these loci was contained within the encoding stage of processing. This conclusion was at variance with some earlier suggestions in the literature (e.g., Theios, 1973, Theios, Smith, Haviland, Traupmann, \& Moy, 1973) which had discussed the effects of probability only with regard to the memory scanning stage. Miller and Pachella's conclusion was problematic, however, since it was based on the close identification of the encoding stage with the factor of stimulus quality. That is, they assumed that the effect of stimulus quality was confined to the encoding processes and did not extend into the memory scan-

The preparation of this paper was supported by NSF Grant BNS-76-82815 to the second author. The authors would like to thank John Jonides and David Meyer for their comments on an earlier draft of the paper. Requests for reprints should be sent to Robert G. Pachella, Human Performance Center, 330 Packard Road, Ann Arbor, Michigan 48104. ning stage. Additive factors logic, by itself, could support either position (or even a combination of the two): Stimulus probability could have its effect early, thus interacting with stimulus quality in the encoding stage; or stimulus quality could have its effect late, interacting with probability in the memory scanning stage (or both factors could affect both stages).

Miller and Pachella (1973) based their conclusion primarily on three previous studies in the literature. Taken together, these studies seemed to indicate that stimulus encoding could be fairly clearly demarcated from memory scanning and that the effect of stimulus quality was confined to the encoding stage of processing. The first study, by Wattenbarger and Pachella (1972), demonstrated that memory load did not affect encoding time. This experiment interspersed choice reaction time trials randomly with standard memory scanning trials, and showed that choice reaction time did not increase as a function of the operative memory load, thus indicating that the effect of memory load was confined to the memory scanning stage. The second, and complementary, study was that of Sternberg (1967), which presented evidence that the effect of stimulus quality was confined to the encoding stage. In this experiment, Sternberg ran memory scanning trials with either clear or degraded stimuli, and, for well-practiced subjects, he obtained equal memory scanning rates for both conditions. Taken together, these two 
studies show the demarcation between encoding and memory scanning. However, Miller and Pachella $(1973,1976)$ were even more influenced by an experiment by Wattenbarger (1970). In this experiment, subjects performed the standard memory scanning task with letters as stimuli. In addition, two variations of the basic task were included. In one condition (the name match condition), the case of the letters presented to the subject was varied, but was irrelevant. That is, if a given letter was included in the memorized set, the subject was to respond "yes" if either the upper- or the lowercase version of the letter appeared as the probe. In the other condition (the physical match condition), case was again varied but was relevant. For example, if a given lowercase letter appeared in the memorized set, the subject responded "yes" only if the lowercase version of the letter appeared as the probe. The uppercase version of the letter was responded to with a "no" response. The basic result of this manipulation led Wattenbarger to conclude that some transformation of the probe stimulus representation had taken place prior to memory scanning, since the slopes of the name-match condition and the control condition were almost identical, while the slope in the physical match condition was nearly twice as great. The normal memory scanning process, whatever its mode of operation, seemed to utilize a representation of the stimulus that was insensitive to physical differences in the probe such as those associated with case differences. Thus, sometime between the onset of the probe and the beginning of memory scanning, a process that removes the effects of physical stimulus quality must take place.

Several theoretical, methodological, and pragmatic problems exist with regard to this line of evidence, however. First, Sternberg (1967) obtained a small interaction between stimulus quality and memory load early in practice. With practice, the interaction disappeared and, furthermore, could not be replicated by Bracey (1969). Nevertheless, some confusion about this interaction still exists (see Sternberg, 1975), particularly since Bracey (1969) had to accept a null hypothesis with data that were not as systematic as those often obtained in memory scanning experiments. Second, the Wattenbarger study, while often cited (e.g., Nickerson, 1972; Sternberg, 1975) has not so far appeared in the archival literature. Thus, investigators have never had access to this complete set of data. Finally, Nickerson (1972) has raised a number of theoretical questions regarding Wattenbarger's interpretation of his data. In particular, he speculated that subjects might have utilized both name and physical codes in some of Wattenbarger's conditions, and noted that his hypothesis would also be consistent with Wattenbarger's data.
As a consequence of these difficulties, this entire line of evidence needs to be reexamined. The present experiments attempt to do this in three ways. First, they present a complete, literal replication of the Wattenbarger experiment. Second, they present a second replication of this experiment under conditions of reduced stimulus quality (reduced contrast in Experiments 1 and 2 and the addition of visual noise in Experiment 2). This manipulation of stimulus quality, together with the relevance of letter case, provides additional evidence about whether or not the effect of stimulus quality is confined to the encoding stage, and also addresses the theoretical questions raised by Nickerson (1972) as to the presence or use of physical codes in any of Wattenbarger's conditions. Finally, the manipulation of stimulus quality in the control or standard memory scanning conditions provides a further replication of the experiments of Bracey (1969) and Sternberg (1967). Taken together, these experiments assess how closely the stimulus encoding stage can be identified with manipulations of stimulus quality under these conditions, and consequently, how clearly interactions of stimulus quality with other factors can be used to localize those factors within the stimulus encoding stage of processing.

\section{EXPERIMENT 1}

\section{Method}

Apparatus. The apparatus, materials, and design of this experiment closely followed that presented by Wattenbarger (1970). A PDP-1 computer controlled the presentation of stimuli and feedback information, and recorded responses and response latencies. The stimuli were consonants $(B, D, F, G, H, M, N$, $Q, R, T)$ presented on a HP 1311A 14" high-speed graphic display. The letters were selected for maximum dissimilarity of their upper- and lowercase representations. Uppercase letters were $1.2 \mathrm{~cm}$ high. Lowercase letters varied from $.6 \mathrm{~cm}(\mathrm{e} . \mathrm{g} ., \mathrm{m})$ to $1.2 \mathrm{~cm}$ (e.g., f), subtending visual angles of .53 and $1.06 \mathrm{deg}$. All letters were $.7 \mathrm{~cm}$ wide. Degradation of the test stimulus was accomplished by lowering a neutral density filter over the CRT, thus producing an approximately 1.5 -log-unit reduction in the figure-ground contrast of the test stimulus. Subjects sat approximately $65 \mathrm{~cm}$ from the screen. Responses were made with the left and right index fingers on a microswitch keyboard located directly in front of the subjects.

Subjects. The subjects were 25 right-handed paid volunteers recruited on the University of Michigan campus.

Design. On each trial, the subject was required to memorize a positive set of from two to five letters, presented sequentially, and respond to a probe letter by indicating whether or not it was a member of the positive set. A "yes" response was made with the right index finger and a "no" response with the left index finger. Half the trials required positive responses and half negative.

There were four types of match conditions. One condition used all uppercase letters (uppercase control). A second condition used all lowercase letters (lowercase control).

The name-match condition used both upper- and lowercase letters, but the case of the letter was irrelevant to the response: Subjects were simultaneously presented with both the upper and lowercase representations of the members of the positive set, with 
the lowercase consistently presented below the uppercase. The probe letter was uppercase for half the trials for each response type and set size, and lowercase for the other half. Thus, it was possible for the subject to make an accurate response on the basis of the name of the probe letter alone.

The physical match condition also used upper- and lowercase letters, but here the case of the letter was relevant to the response: Subjects were presented with either the upper- or lowercase representation of each member of the positive set. Again, the probe letter was uppercase on half of the trials for each response type and set size and lowercase for the other half. As nearly as possible, each trial contained equal numbers of upper- and lowercase positive set size members. The uppercase representations predominated on half the trials for set sizes 3 and 5 . In this condition, there were two types of negative trials: A case-mismatch (CM) negative, where the name of the probe letter matched a member of the positive set but the case differed, and a pure negative response where the name of the probe letter was not contained in the positive set. Half of the negative responses (.25 of the total) were $\mathrm{CM}$ negative, and half were pure negative.

Procedure. Each subject served in two sessions of approximately $2 \mathrm{~h}$ each on successive days. Each session consisted of four practice blocks of 16 trials followed by six experimental blocks of 52 trials. The experimental blocks were defined by the orthogonal combination of three type of match conditions (control, name identity, and physical identity) and two levels of stimulus contrast. The practice blocks utilized the control condition that was not being included in the experimental blocks for the particular subject. Within a block, equal numbers of trials were presented randomly for four sizes of the positive set. Blocks of trials alternated between normal and degraded presentation. The orders of type of match and stimulus contrast conditions were counterbalanced across subjects. The order of presentation of the degraded blocks was reversed for subjects in the second session.

Each positive set member was presented on the lower half of the screen for $1,000 \mathrm{msec}$ with a 500 -msec delay between presentations. This was followed by a plus sign indicating a fixation point on the upper half of the screen. The fixation point was terminated after $1,500 \mathrm{msec}$. Following a delay of $500 \mathrm{msec}$, the probe letter was presented until either the subject made a response or $3 \mathrm{sec}$ had elapsed. Feedback was presented in the form of a plus sign for correct responses and a minus sign for incorrect responses. The feedback signal was presented on the lower half of the screen for $500 \mathrm{msec}$ and served as the fixation point for the presentation of the positive set for the next trial. The intertrial interval was $500 \mathrm{msec}$. Subjects were allowed to interrupt the trial sequence briefly and were encouraged to do so if they felt fatigued or confused after making an error. After every two blocks of trials, the subjects were given a S-min rest.

A trial was considered an error if the subject made a wrong response or exceeded a criterion time of $3 \mathrm{sec}$. Error trials were repeated later in the block of trials until a correct response was made. Any subject who made more than 20 errors in the $\mathbf{2 8 8}$ trials of the six experimental blocks in either session was discarded. One of the original 24 subjects was excluded by this criterion; a new subject was run to replace him.

\section{Results and Discussion}

Trials from the practice blocks at the beginning of each session, the first four trials from each experimental block, and all error trials were excluded from the data analysis. The subsequent analyses were done on 48 correct trials for each of six experimental blocks per session for 24 subjects.

An analysis of variance performed on the control conditions with type of control (upper- vs. lowercase) as a between-subjects variable revealed no sig- nificant difference between the control conditions $[\mathrm{F}(1,20)=.6, \mathrm{MSe}=303,253]$. Therefore, the data from the two conditions were combined and treated as a single condition (control).

For each subject, a mean was calculated for each combination of session, type of match condition, stimulus contrast, positive set size, and response type. A linear function relating RT to set size was calculated by the least squares method for each subject for each of 24 cells in the design. Subsequent tests were performed on the resulting slopes and intercepts.

An analysis of variance performed on the intercepts revealed a significant main effect of type of match condition $[\mathrm{F}(2,24)=5.23, \mathrm{p}<.05, \mathrm{MSe}=$ $48,959]$, as did the analysis of the slopes $[F(2,24)=$ $51.79, \mathrm{p}<.01, \mathrm{MSe}=3,606]$. The effect of type of match condition for both main effects is due to the physical identity condition. Newman-Keuls tests revealed that the physical identity condition was significantly different in both slope and intercept from the control and name identity conditions which did not differ from each other. These results are shown in Figure 1.

The slopes and intercepts of the functions for the name identity and control conditions are nearly identical. The slopes and intercepts obtained in the high-contrast test stimulus condition in this experiment nicely replicate those obtained by Wattenbarger (1970) and agree well with those found in other memory scanning experiments using letters as stimuli. In the name identity condition, subjects were presented with twice as many visual images as in the control condition. Since case was irrelevant, however, both representations of the letter could be encoded with one name. It is reasonable to assume that in both the name identity and control conditions the subjects were identifying the stimulus and

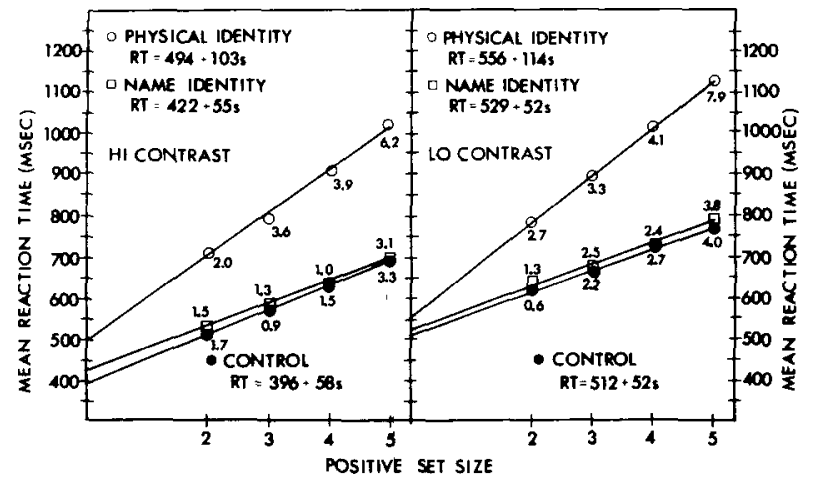

Figure 1. Reaction time as a function of set size and type of match for two levels of stimulus contrast in Experiment 1. The least squares fit for the linear function of reaction time as a function of set size accounted for better than $99 \%$ of the variance in all conditions. Numbers adjacent to each point indicate the percentage of error for each condition. 
using some abstract representation for the comparison. The greater intercept and slope for the physical identity condition could be the result of subjects' having to use a more complicated representation for this condition. They were required to encode both the name of the letter and some indication of its case. Memory for the letters would involve more information and result in a longer scanning time per item for members of the positive set (Cavanaugh, 1972).

The analysis of variance performed on the intercepts also revealed significant main effects of session $[F(1,12)=10.99, p<.01, \mathrm{MSe}=68,168]$, stimulus contrast $[\mathrm{F}(1,12)=33.79, \mathrm{p}<.01, \mathrm{MSe}=38,847]$, and response type $[\mathrm{F}(1,12)=33.95, \mathrm{p}<.01, \mathrm{MSe}=$ $18,416]$, and a significant three-way interaction between session, type of match condition, and stimulus contrast $[F(2,24)=3.88, p<.05$, MSe $=16,085]$.

The analysis of variance performed on the slopes also revealed a significant interaction between stimulus contrast and type of match condition $[F(2,24)$ $=3.47, \mathrm{p}<.05$, MSe $=1,176]$. Tests of the simple main effects of stimulus contrast at each condition level revealed that the effect of degradation on the slopes of the functions was significant only for the physical identity condition $[F(1,24)=4.85, p<.05$, MSe $=1,176]$. The additivity of stimulus clarity and set size in the control and name identity conditions is illustrated by the small variation in the slopes of the functions, as noted in Figure 1. The additivity for these two conditions provides further support for the conclusion that an abstract code is the representation used in the comparison stage.

The control conditions in the present experiment provided an opportunity to replicate Sternberg's (1967) results. These data, like those of Bracey, provide no evidence for interaction between stimulus contrast and set size in the control condition. The size of the effect of stimulus contrast on the intercepts of the RT functions in the name identity and control conditions is approximately $100 \mathrm{msec}$, compared to the 60 -msec effect obtained by Sternberg. The degradation manipulation used in this experiment should therefore have been large enough to strain a filtering operation in the encoding stage and yield an interaction between stimulus contrast and set size if a physical code was being used in the memory scanning process. This noninteraction, together with the identity of the slopes for the name and control conditions, thus argues strongly for the use of some transformed abstract representation from which physical characteristics of the stimulus (i.e., case and contrast) have been removed.

The nonadditivity of stimulus quality and set size in the physical identity condition allows the tentative hypothesis that subjects might be utilizing some physical code in this condition. This conclusion, however, would be based on a very small interaction (see Figure 2). Furthermore, it is inconsistent with two other aspects of the data: First, the analysis of variance on the intercepts revealed a significant twoway interaction between type of match condition and response type $[\mathrm{F}(2,24)=5.49, \mathrm{p}<.025, \mathrm{MSe}=$ $23,865]$. The interaction resulted primarily from a greater difference between the positive and combined negative response types in the physical identity condition than was found for the positive and negative responses in the name identity or control conditions. Analyses of data from the physical identity condition alone allowed the negative functions (i.e., case mismatch and pure negative responses) for that condition to be analyzed separately. Analysis of the intercepts for the physical identity condition revealed a significant main effect of trial type $[F(2,24)=$ 14.49, $\mathrm{p}<.01, \mathrm{MSe}=57,818]$. A Newman-Keuls test indicated that the main effect was due to the $\mathrm{CM}$ negative trials, which had significantly greater intercepts than the pure negative and positive trials, which did not differ from each other. The intercept differences for the different trial types for the physical identity condition are shown in Figure 3. The intercept difference replicated that reported by Wattenbarger (1970), and supports a name represen-

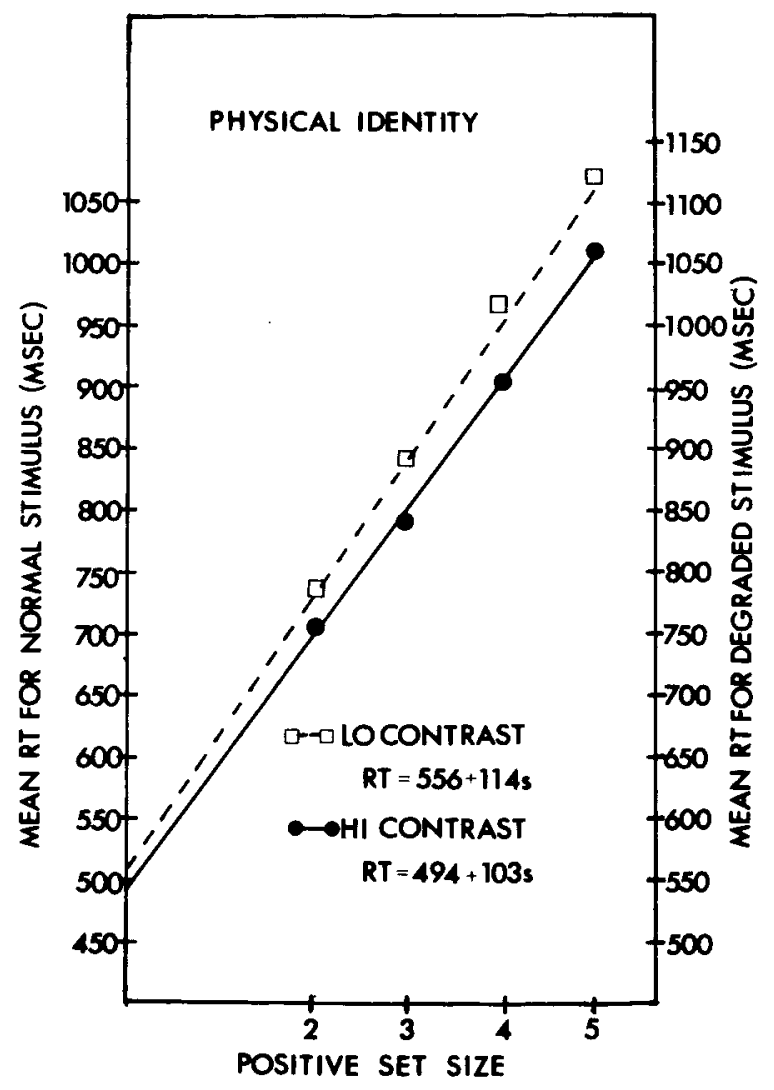

Figure 2. Reaction time as a function of set size and stimulus contrast for the physical identity comparison condition in Experiment 1. The scale for the normal stimulus conditions has been shifted upward. 


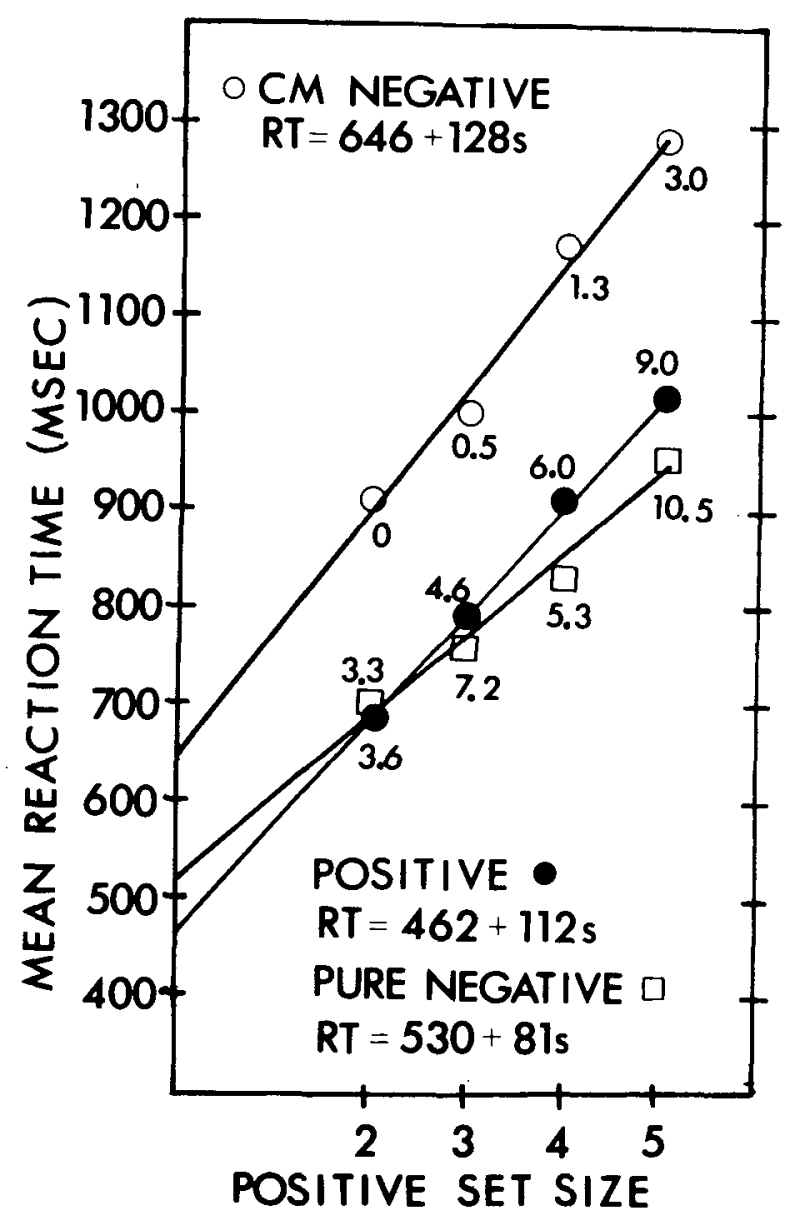

Figure 3. Reaction time as a function of set size and trial type for the physical identity comparison condition in Experiment 1. The least squares fit for the linear function relating reaction time to set size accounted for better than $98 \%$ of the variance in all conditions. Numbers adjacent to each point indicate the percentage of error for each condition.

tation hypothesis for the physical identity condition. If a physical code is used in memory scanning, the $\mathrm{CM}$ and pure negative trials should be quite similar. However, if a complicated name representation that includes some indication of case is used, then the $\mathrm{CM}$ negative trials will always involve one comparison between two representations that include the same name and differ only in case marking. This similarity would cause interference that would show up as an intercept effect, since it only happens once on each $\mathrm{CM}$ negative trial.

Second, there is also a significant effect of trial type on the slopes of the reaction time functions for the physical identity condition $[F(2,24)=7.37$, $\mathrm{p}<.01$, MSe $=7,312$ ]. A Newman-Keuls test indicated that the slope of the function for the pure negative trials was significantly lower than the slopes for the positive and CM negative trials which did not differ from each other. The slope differences of the functions shown in Figure 3 are in the same direction as the nonsignificant differences found by
Wattenbarger (1970). His reported slopes were 88, 97 , and $102 \mathrm{msec}$ for the pure negative, positive, and $\mathrm{CM}$ negative trials, respectively. The differences in slopes for the various trial types can be explained if one assumes that the physical identity condition causes differential uncertainty on the part of the subjects so that they rescan memory to check on matches or mismatches before making a response. On both $\mathrm{CM}$ negative and positive trials, a name match has been found and the response decision must therefore be determined by case marking alone, whereas a name mismatch leads directly to a negative response.

Alternatively, the memory set representations may be identical for the name identity and physical identity matches, and the type of match instructions may determine the nature of the probe representation. For example, the memory set may always contain both name and case information. The physical identity condition may involve dual representations of the probe stimulus: an abstract, normalized physical code as well as a name code. Under nameidentity instructions, the subject would compare a name code of the stimulus against the memory set. Under physical identity instructions, the subject would first compare a name code with the memory set in order to reject name-different codes. If a name match was found, the subject would then compare the abstract physical code against the memory set. Name identity matches would require one scan, while physical identity matches would require two. The pure negative physical identity trials would require only one scan, hence the lower slope of RT across set size for these trials. ${ }^{1}$ As noted earlier, this account is similar to that proposed by Nickerson (1972) in discussing Wattenbarger's results.

Thus, Experiment 1 replicates all of the essential findings of the Wattenbarger (1970) study. Furthermore, the manipulation of contrast allows additional convergence on Wattenbarger's conclusions while at the same time providing a replication of Sternberg's (1967) study with regard to the control conditions. This latter replication is in agreement with Sternberg's findings for practiced subjects and in complete agreement with Bracey's (1969) findings.

\section{EXPERIMENT 2}

The primary purpose of the second experiment was to investigate the interaction between type of match condition and stimulus contrast found in the first experiment. This interaction needs to be investigated because of its importance for determining the form of the internal representation used by subjects in performing physical identity classifications. The highcontrast test-letter data indicate that perhaps a more elaborate name code is used in the physical identity condition. However, the effect of degradation on this 
condition implies that a more complicated explanation may be needed; one that would incorporate a physical comparison process by the subject and still be consistent with other aspects of the data.

The second experiment was also designed to test the effects of degradation by visual noise on the name identity and physical identity conditions. The visual noise mask more closely resembles the checkerboard pattern degradation used by Sternberg (1967) than the contrast reduction used in the first experiment. Moreover, recent research by Miller (1976) suggests that the visual noise mask might affect a different substage of encoding than that affected by reducing the contrast of the test stimulus. Specifically, Miller's (1976) data have suggested that contrast reduction affects a very early substage of encoding where the figure to be identified is separated from the background, while visual noise affects a somewhat latter substage prior to the identification of the letter.

\section{Method}

The method of this experiment was nearly identical to that of Experiment 1. The same stimuli and apparatus were used. Seventeen right-handed subjects were recruited on the University of Michigan campus. None of the subjects had participated in the first experiment. Subjects were run in two identical sessions of approximately $2 \mathrm{~h}$ each. Only the name identity and physical identity conditions of the first experiment were used. Each condition occurred with three levels of stimulus degradation: a normal condition involving no degradation, the contrast reduction condition of the first experiment, and degradation via a visual noise pattern superimposed over the test letter. Noise patterns were constructed following the procedure of Miller (1976). They were composed of 144 dots scattered over a square area $3 \mathrm{~cm}$ on a side. The patterns were generated by treating the area as a 12 by 12 grid of smaller squares. On each trial, one dot was randomly placed within each of the small square areas of the grid. Thus, the overall distribution of dots in the pattern was constant from trial to trial, though the exact placement varied. Each dot was generated by illuminating one raster unit on the screen. The screen grain is 72 raster units per vertical inch and 64 raster units per horizontal inch. The orders of presentation of type of match condition and stimulus degradation were counterbalanced across subjects.

Each of the first four blocks of a session consisted of 32 trials using uppercase letlers for practice. The first block was the normal condition, the second used contrast reduction, the third used random visual noise for degradation, and the fourth practice block repeated the normal condition. This was followed by six experimental blocks of $\mathbf{4 8}$ trials. Only positive set sizes of two and four were used in this experiment. Counterbalancing of response type, probe case, and set size was the same as in Experiment 1, as were the trial-by-trial procedure and the treatment of error trials. Any subject who made more than 20 errors in the 240 trials of the six experimental blocks in either session was discarded. Five subjects were excluded by the error criterion, and new subjects were run to replace them.

\section{Results and Discussion}

Trials from the practice blocks at the beginning of each session, the first eight trials from each experimental block, and all error trials were excluded from the data analysis. The subsequent analyses were done on the 40 correct trials for each of the six experimental blocks per session for 12 subjects.
For each subject, a mean was calculated for each combination of session, type of match condition, stimulus degradation condition, positive set size, and response type. A straight line connecting the RT values for the two values of set size and extended to the ordinate was fit for each subject for each of 24 cells in the design. Analyses of variance were performed on the resulting slopes and intercepts.

An analysis of variance performed on the slopes revealed a significant main effect of type of match condition $[F(1,10)=54.39, p<.01, \mathrm{MSe}=7,921]$. The main effect, illustrated in Figure 4, replicates that of Experiment 1. The slope for the physical identity condition is significantly greater than the slope for the name identity condition. The intercept difference between the two conditions found in the first experiment was not replicated here $[\mathrm{F}(1,10)=.84$, $\mathrm{MSe}=68,834]$, although it is in the same direction as that found in the first experiment.

The interaction between type of match condition and stimulus degradation was also significant in the analysis of the slopes $[\mathrm{F}(2,20)=3.59, \mathrm{p}<.05$, MSe $=2,631]$. The interaction is shown in Figure 5. This interaction was decomposed into orthogonal contrasts. The effect of the random noise degradation vs. the other two degradation conditions was the only significant posterior contrast $[\mathrm{F}(2,20)=3.41, \mathrm{p}<$ .05 , MSe $=2,631]$. Thus, it should be noted that the effect of degradation by contrast reduction on the

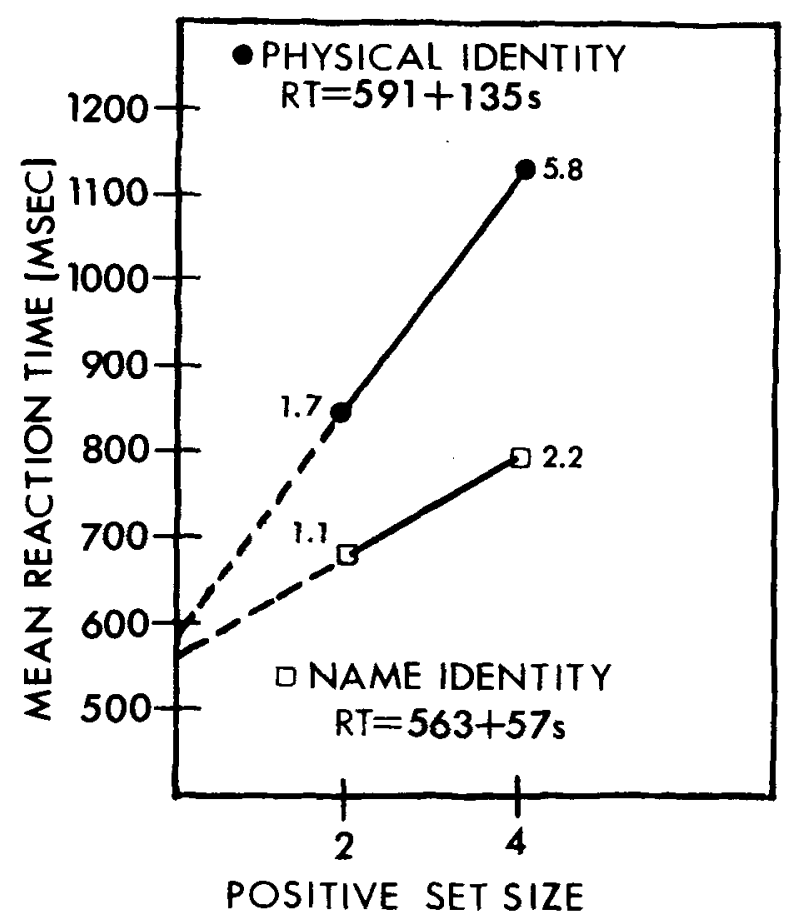

Figure 4. Reaction time as a function of set size for the name and physical identity conditions of Experiment 2. 


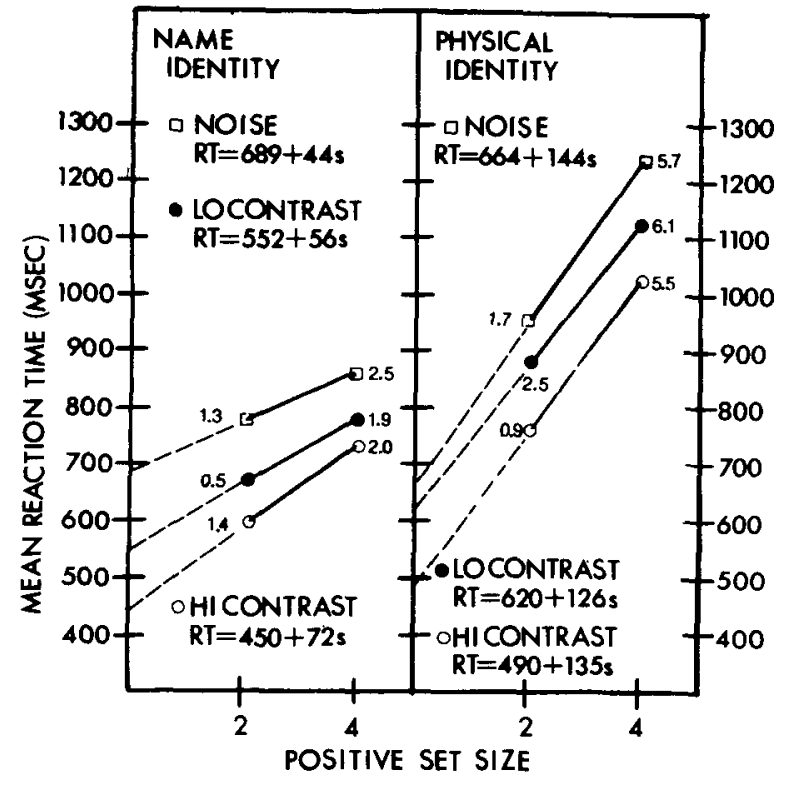

Figure 5. Reaction time as a function of set size and three levels of stimulus degradation for the two type-of-match conditions in Experiment 2. Numbers adjacent to each point indicate the percentage of error for each condition.

slope of the physical identity condition obtained in Experiment 1 was not replicated in the second experiment. Furthermore, an analysis of variance on the slopes for the physical identity condition alone revealed no significant effect of stimulus degradation $[F(2,20)=.82$, MSe $=10,099]$. Thus, the interaction of type of match condition and degradation seems to be due to the underadditivity of stimulus noise on the slopes of the functions in the name identity condition. This underadditivity is heightened by the abnormally high slope $(72 \mathrm{msec})$ for the highcontrast test stimulus condition.

The analysis of variance performed on the slopes for the physical identity condition alone revealed a significant effect of trial type (i.e., positive vs. pure negative vs. $\mathrm{CM}$ negative) $[\mathrm{F}(2,20)=11.0, \mathrm{p}<.01$, $\mathrm{MSe}=13,350]$. A Newman-Keuls test indicated that the slope for the pure negative trials was significantly less than the slopes for the yes and CM negative trials, which did not differ from each other. This result, illustrated in Figure 6, replicates the slope effect found in Experiment 1. No other slope effects were significant in this experiment.

Analysis of variance performed on the intercepts also replicated the results for the first experiment. There were significant main effects of session $[F(1,10)$ $=13.26, \mathrm{p}<.01$, MSe $=43,630$ ), stimulus degradation $[\mathrm{F}(2,20)=16.69, \mathrm{p}<.01, \mathrm{MSe}=61,922]$, and response type $[\mathrm{F}(1,10)=23.51, \mathrm{p}<.01, \mathrm{MSe}=$ 47,299 ]. The trial type factor was also significant in the intercept analysis on data from the physical identity condition alone $\{\mathrm{F}(2,20)=8.14, \mathrm{p}<.01$, MSe $=104,253]$. As in Experiment 1, the effect was due to the CM negative trials, which were significantly different from the yes and pure negative trials.

The results of Experiment 2 thus far provide a replication of all of the essential elements of the first experiment and clear support for the hypothesis that an abstract code is the internal representation used in the memory scanning stage when both name and physical identity matches are required. We have failed, however, to replicate the small interaction between stimulus contrast and type of match condition found in Experiment 1.

An analysis of variance was also performed on the individual subject means for the 48 cells of the experimental design ( 2 match conditions by 2 responses by 2 sessions by 2 set sizes by 3 modes of degradation). This analysis produced a number of significant effects that were consistent with the previous analyses, but additional findings were also demonstrated. One of these results was a significant two-way interaction between mode of degradation and response type $[F(2,20)=5.41, p<.025, \mathrm{MSe}=50,360]$, which is shown in Figure 7. In general, negative responses were slower than positive responses, and the size of the difference was about the same for both the low- and high-contrast conditions. However, the difference between the positive and negative

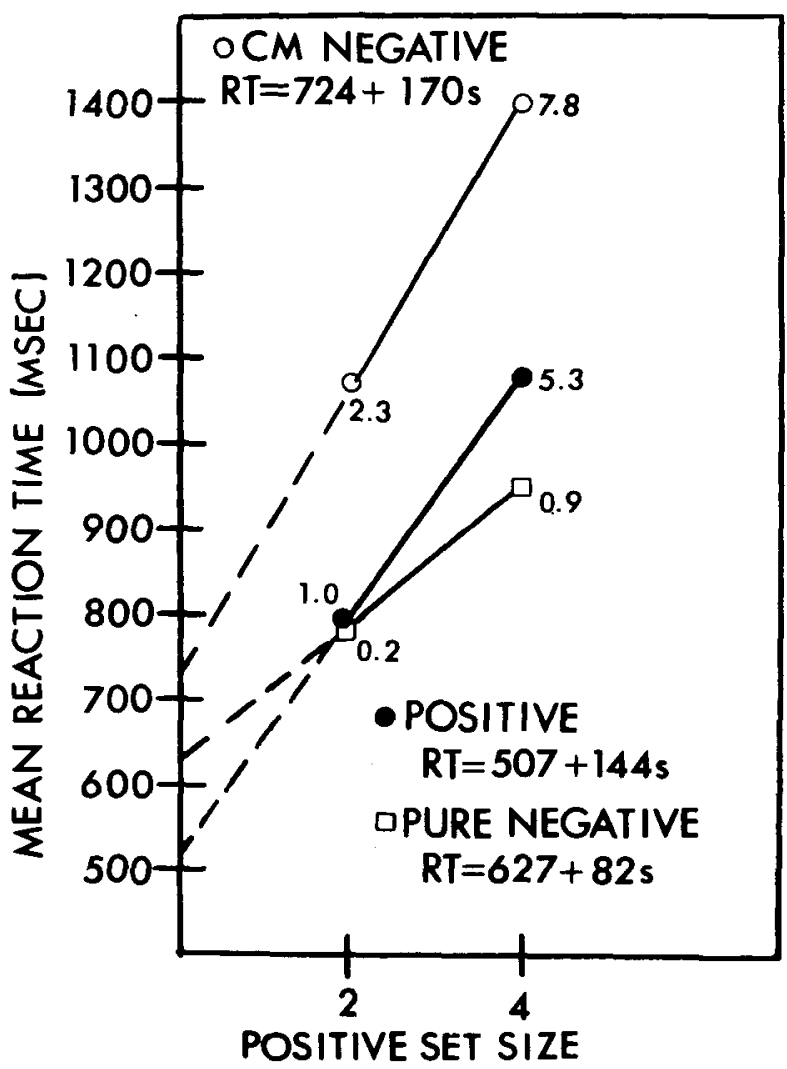

Figure 6. Reaction time as a function of set size and trial type for the physical identity condition in Experiment 2. Numbers adjacent to each point indicate the percentage of error for each condition. 


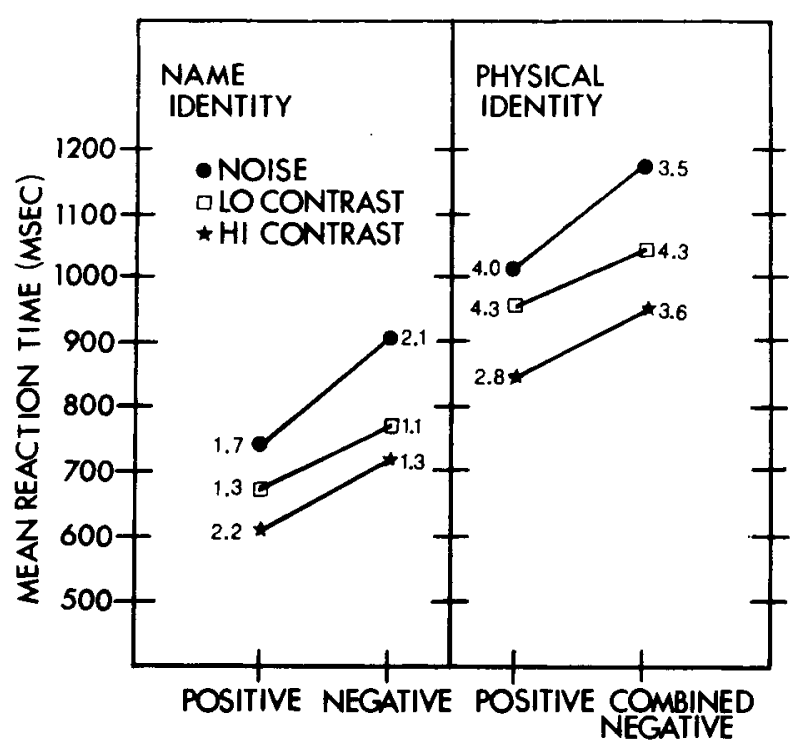

RESPONSE TYPE

Figure 7. Reaction time as a function of response type and three levels of stimulus degradation for two types of match in Experiment 2. Numbers adjacent to each point indicate the percentage of error for each condition.

responses was accentuated in the presence of visual noise.

This last result is consistent with our general hypothesis that an abstract representation of the name of the letter is extracted from the stimulus trace, although it is more speculative, and it is also consistent with Miller's (1976) finding that contrast and noise affect different substages of encoding. The tentative explanation of this effect, based on the idea that its locus is in the letter identification process, is that the prior encoding of the positive set enhances the extraction of features of the members of the positive set when they occur as probe items. Since this enhancement can take place only on positive trials, and since the size of the effect is the same for both name and physical matches, and since the presence of noise does not affect the slope of the memory scanning process, it seems reasonable to conclude that feature extraction takes place before memory scanning. Furthermore, Miller (1976) has recently shown that it is feature extraction, as opposed to figure ground separation, that is affected by visual noise, while the latter process is affected by contrast reduction. Thus, this evidence, while not as central to our basic arguments as some of the earlier results, is at least consistent with the notion that identification takes place before memory scanning.

\section{GENERAL DISCUSSION}

The experiments reported here provide evidence for the hypothesis that stimulus letters are transformed through encoding processes into an abstract internal representation. It is this abstract internal representation that the subject uses for memory comparisons in the memory scanning stage. Several types of evidence converge on this conclusion. First, the slope of the function relating $\mathrm{RT}$ to set size for physical identity comparisons is greater than the slope for name identity and control comparisons. This is true even though there potentially are twice as many visual representations in the name identity condition. The physical identity comparisons involve more complicated abstract representations and, hence, more scanning time per item for members of the positive set (cf. Cavanaugh, 1972). The slope and intercept differences found for the three comparison types in the physical identity condition also lend support to this conclusion. If some sort of visual representation is used in the memory comparison stage, then one would expect the pure negative and CM negative trials to be identical. However, these trials are different, due perhaps to confusion and uncertainty when a name match is found and case markers differ.

Of most importance with regard to Nickerson's (1972) objections, reducing the contrast of the test stimulus against its background or superimposing a visual noise pattern over a test stimulus adds a constant amount of response time across set size. The only instance in which this was not the case was in the small interaction found in the first experiment between stimulus contrast and set size with physical identity comparisons. However, this interaction was not replicated in the second experiment. Thus, it seems unlikely that simple physical codes are used in memory scanning, since, in that case, reduced stimulus contrast would slow the rate of scanning. Finally, the control conditions in the first experiment were designed to replicate Sternberg's (1967) results. This replication condition demonstrated clear additivity between stimulus contrast manipulations and set size, and this result was replicated for the name match condition of Experiment 2. This indicates that the effects of degradation are removed prior to the memory comparison stage; an expected result if an abstract representation is used in the comparison process.

All of these data taken together supply much information about the now commonly used memory scanning task, and it should be noted that virtually all of it is independent of many of the competing models of the memory scanning subprocess itself. It has been our purpose, more importantly, to demonstrate that regardless of what goes on in the memory dependent stage, the transformation of the probe character is complete before scanning (or whatever) takes place. Thus, the effects of stimulus quality are confined to the encoding processes and factors that interact with stimulus quality, such as stimulus probability, are likely also to have a locus of effect within the encoding stage.

Three caveats need to be entered and noted in conclusion. First, while the foregoing has attempted to 
argue for and demonstrate a clear demarcation between the stimulus encoding and memory scanning process in the simple memory scanning task, we do not wish to leave the impression that this argument leads to the conclusion that the physical code of the stimulus is necessarily terminated by the transformation to an abstract representation during encoding. As Posner and Warren (1972) have pointed out, it is entirely possible that both codes can exist simultaneously and be utilized in appropriate circumstances. Our particular point has been to show that the critical processing path during the course of the standard memory scanning task involves the particular process of abstraction most classically associated with the concept of encoding. The factor of stimulus quality can be used to isolate important and meaningful characteristics of this process. Clearly, theoretical confusion can arise if basic modifications to this task are developed that require the use of the physical code of the stimulus. Interestingly enough, however, we have demonstrated that requiring the subject to make physical matches during memory scanning seems not to be one of those circumstances; rather, the subject seems to still rely on some form of complex abstract code.

Second, some care must be taken before the conclusions of the present paper can be generalized beyond the specifics of the present methodology. Of perhaps greatest methodological significance is the duration of the interval between the presentation of the positive set and the probe stimulus. In the present experiments, this interval was $2 \mathrm{sec}$. Evidence by Thorson, Hochhaus, and Stanners (1976) indicates that the conversion from a visual to a nonvisual code for letter stimuli takes on the order of about $1 \mathrm{sec}$. Consequently, variations of the memory scanning task with very short intervals between the end of the positive set and the probe item could conceivably disrupt the discrete nature of the encoding process that has been argued for in the present experiments.

Third, the results of the present experiments can be generalized most widely under the assumption that the varied set procedure and the fixed set procedure of memory scanning involve the same mode of probe stimulus encoding during the test trial. The present experiments have utilized the former (varied set) procedure in which a different memory set is presented to the subject on every trial. The latter (fixed set) procedure, in which the same memory set is used for an entire block of trials, was used by Miller and Pachella $(1973,1976)$. It seems reasonable that, with a long enough interval between the presentation of the memory set and the probe, as noted in the last paragraph, the two procedures would yield similar probe encoding functions. Others (notably Sternberg, 1975) have also argued for this possibility. Nevertheless, since other researchers have found differences between the procedures (e.g., Schneider \& Shiffrin, 1977), such assumptions clearly need further empirical justification. In the meantime, the procedures described in the present paper seem to provide evidence that discrete and well-defined encoding operations can be isolated with the manipulation of stimulus quality.

\section{REFERENCES}

Bracey, G. Two operations in character recognition: A partial replication. Perception \& Psychophysics, 1969, 6, 357-360.

Cavanaugh, J. P. Relation between immediate memory span and the memory search rate. Psychological Review, 1972, 79, 525-530.

MilleR, J. O. Effects of stimulus probability on encoding mechanisms in information-processing tasks. Unpublished $\mathrm{PhD}$ thesis, University of Michigan, 1976.

Miller, J. O., \& Pachella, R. G. Locus of the stimulus probability effect. Journal of Experimental Psychology, 1973, 101, 227-231.

Miller, J. O., \& Pachella, R. G. Encoding processes in memory scanning tasks. Memory \& Cognition, 1976, 4, 501-506.

NiCKERSON, R. S. Binary classification reaction time: A review of some studies of human information-processing capabilities. Psychonomic Monograph Supplements, 1972, 4(17, Whole No. 65), 275-318.

Posner, M. I., \& Warren, R. E. Traces, concepts, and conscious constructions. In A. W. Melton \& E. Martin (Eds.), Coding processes in human memory. New York: Wiley, 1972.

Schneider, W., \& Shiffrin, R. M. Controlled and automatic human information processing: I. Detection, search, and attention. Psychological Review, 1977, 84, 1-66.

Stanovich, K. E., \& Pachella, R. G. Encoding, stimulusresponse compatibility, and stages of processing. Journal of Experimental Psychology: Human Perception and Performance, $1977,3,411-421$.

STERnBerg, S. Two operations in character recognition: Some evidence from reaction-time measurements. Perception \& Psychophysics, 1967, 2, 45-53.

STERnBerG, S. The discovery of processing stages: Extensions of Donder's method. In W. G. Koster (Ed.), Attention and performance II. Acta Psychologica, 1969, 30, 276-315.

Sternberg, S. Memory scanning: New findings and current controversies. Quarterly Journal of Experimental Psychology, $1975,27,1-32$.

Theios, J. Reaction time measurements in the study of memory processes: Theory and data. In G. Bower (Ed.), The psychology of learning and motivation: Advances in research and theory (Vol. 7). New York: Academic Press, 1973.

Theios, J., Smith, P. G., Haviland, S. E., Traupmann, J., \& Moy, M. C. Memory scanning as a serial, self-terminating process. Journal of Experimental Psychology, 1973, 97, 323-336.

Thorson, G., Hochhaus, L., \& Stanners, R. F. Temporal changes in visual and acoustic codes in a letter matching task. Perception \& Psychophysics, 1976, 19, 346-348.

WATTENBARGER, B. L. The representation of the stimulus in character classification. Unpublished PhD thesis, University of Michigan, 1970.

Wattenbarger, B. L., \& Pachella, R. G. The effect of memory load on reaction time in character classification. Perception \& Psychophysics, 1972, 12, 100-102.

\section{NOTE}

1. This alternative explanation was suggested by an anonymous reviewer.

(Received for publication September 27, 1979; revision accepted December 3, 1979.) 